\title{
Nuclear Force from Lattice QCD
}

\section{Noriyoshi ISHII*}

Department of Physics, University of Tokyo, Tokyo 113-0033, JAPAN

E-mail: ishii@rarfaxp.riken.jp

\section{Sinya AOKI}

Graduate School of Pure and Applied Science, University of Tsukuba, Tukuba 305-8571,

Ibaraki, JAPAN

E-mail: saoki@het.ph.tsukuba.ac.jp

\section{Tetsuo HATSUDA}

Department of Physics, University of Tokyo, Tokyo 113-0033, JAPAN

E-mail: hat suda@phys.s.u-tokyo.ac.jp

The first lattice QCD result on the nuclear force (the NN potential) is presented in the quenched level. The standard Wilson gauge action and the standard Wilson quark action are employed on the lattice of the size $16^{3} \times 24$ with the gauge coupling $\beta=5.7$ and the hopping parameter $\kappa=0.1665$. To obtain the NN potential, we adopt a method recently proposed by CP-PACS collaboration to study the $\pi \pi$ scattering phase shift. It turns out that this method provides the NN potentials which are faithful to those obtained in the analysis of NN scattering data. By identifying the equal-time Bethe-Salpeter wave function with the Schrödinger wave function for the two nucleon system, the NN potential is reconstructed so that the wave function satisfies the time-independent Schrödinger equation. In this report, we restrict ourselves to the $J^{P}=0^{+}$and $I=1$ channel, which enables us to pick up unambiguously the "central" NN potential $V_{\text {central }}(r)$. The resulting potential is seen to posses a clear repulsive core of about $500 \mathrm{MeV}$ at short distance $(r \lesssim 0.5 \mathrm{fm}$ ). Although the attraction in the intermediate and long distance regions is still missing in the present lattice set-up, our method is appeared to be quite promising in reconstructing the NN potential with lattice QCD.

XXIVth International Symposium on Lattice Field Theory

July 23-28, 2006

Tucson, Arizona, USA

\footnotetext{
${ }^{*}$ Speaker.
} 


\section{Introduction}

The nuclear force (the NN potential) serves as one of the most important building blocks in nuclear physics. Its attractive part in the intermediate distance region plays an essential role to bind nucleons in nuclei. On the other hand, its strong repulsive core at short distance ensures the stability of heavy nuclei by preventing them from collapsing and leads to the celebrated saturation phenomena of nuclear matter. Importance of the nuclear force goes even beyond the nuclear structure: It can have a great influence on the physics of compact stars and the supernova explosion through the equation of state at high baryon densities.

So far, enormous effort has been devoted to understand the nuclear force theoretically [1]. The long distance region $(r \gtrsim 2 \mathrm{fm})$ of the nuclear force is dominated by the exchange of a single pion, i.e., the lightest elementary excitation in the QCD spectrum. The intermediate distance region $(1 \lesssim r \lesssim 2 \mathrm{fm}$ ) receives a significant contribution from multi-pion exchanges and from heavy meson exchanges such as $\rho, \omega$, and " $\sigma$ ". The understanding of the short distance region $(r \lesssim 1 \mathrm{fm})$ is most retarded, but it is believed to be intimately related to the quark-gluon structure of the nucleons.

Hence, the first principle QCD calculation of the nuclear force (in particular the short distance region) has been desired for a long time. Recently, a method attempting to obtain the origin of the repulsive core on the lattice was proposed, where the nucleon is assumed to be composed of heavy-light-light quarks so that one can define the relative distance between the nucleons in terms of the distance between the heavy quarks [2]. (The same technique was also used in Ref. [3].) In this report, we introduce a totally different approach to the NN potential on the basis of a method recently proposed by CP-PACS collaboration in the context of $\pi \pi$ scattering phase shift [5]. For simplicity, we confine ourselves to the channel $J^{P}=0^{+}$and $I=1$, which makes us possible to obtain unambiguously the so-called central potential $V_{\text {central }}(r)$. First, we construct the equal-time Bethe-Salpeter (BS) wave function for the NN system. It is then identified with the non-relativistic Schrödinger wave function for two nucleons interacting at low energies. $V_{\text {central }}(r)$ is reconstructed so that the wave function satisfies the non-relativistic Schrödinger equation. In this way, we do not have to introduce heavy quarks to define the relative distance unlike the method propose before.

The reconstructed $V_{\text {central }}(r)$ has a repulsive core of about $0.5 \mathrm{GeV}$ in the short distance region ( $r \lesssim 0.5 \mathrm{fm}$ ). On the other hand, the attraction in the intermediate and long distance regions is still missing, which may be attributed to combined artifacts of the heavy pion mass $\left(m_{\pi} \sim 0.5 \mathrm{GeV}\right)$, the small spatial volume $(L \sim 2.2 \mathrm{fm})$ and statistics.

\section{The formalism}

We consider the equal-time Bethe-Salpeter (BS) wave function $\phi(\vec{x} ; k)$ for NN system in the $J^{P}=0^{+}$and $I=1$ channel as

$$
\begin{aligned}
\phi(\vec{x} ; k) & \equiv \frac{1}{24} \sum_{R \in O} \frac{1}{L^{3}} \sum_{\vec{X}}\left(\sigma_{2}\right)_{\alpha \beta}\left\langle 0\left|p_{\alpha}(R \cdot \vec{x}+\vec{X}) n_{\beta}(\vec{X})\right| p n ; k\right\rangle, \\
p_{\alpha}(x) & \equiv \varepsilon_{a b c}\left(u_{a}^{T} C \gamma_{5} d_{b}\right) u_{c, \alpha} \\
n_{\beta}(x) & \equiv \varepsilon_{a b c}\left(u_{a}^{T} C \gamma_{5} d_{b}\right) d_{c, \beta},
\end{aligned}
$$

where $a, b$ and $c$ denote the color indices, $\alpha$ and $\beta$ the Dirac indices, and $C \equiv \gamma_{4} \gamma_{2}$ the charge conjugation matrix. $p_{\alpha}(x)$ and $n_{\beta}(y)$ denote composite bispinor fields for proton and neutron, 
which are represented with Dirac's convention. $\vec{x}$ plays the role of the spatial separation of the proton from the neutron. The summation over $R$ is performed for the cubic transformation group $O$ to pick up the s-wave orbital contribution of NN system. Note that, due to the parity selection rule, only s-wave contribution is allowed for $J^{P}=0^{+}$. The spinors of the proton and the neutron are combined into scalar in the non-relativistic manner with $\left(\sigma_{2}\right)_{\alpha \beta}$. Since we are interested in the significantly low energy region, the lower components of the nucleon bispinors, i.e., $p_{\alpha}$ and $n_{\beta}$, are negligible. Since this wave function is symmetric in orbit and anti-symmetric in spin configuration, it has to be symmetric in iso-spin space due to the Pauli statistics, i.e., this wave function is iso-vector. The summation over $\vec{X}$ is performed to project the total spatial momentum to zero, i.e., $\vec{P}=\overrightarrow{0}$. The Lüscher's $k$ is introduced as a relative momentum outside the range of the NN interaction, playing the role of "asymptotic relative momentum" between the proton and the neutron in the scattering theory in the infinite volume limit.

The BS wave function Eq. (2.1) is obtained, in the lattice QCD formulation, from the large $t$ behavior of the four point nucleon correlator as

$$
\begin{aligned}
F_{N N}\left(\vec{x}, \vec{y}, t ; t_{0}\right) & \equiv\left\langle 0\left|p_{\alpha}(\vec{x}, t) n_{\beta}(\vec{y}, t) \bar{p}_{\alpha^{\prime}}\left(\overrightarrow{0}, t_{0}\right) \bar{n}_{\beta^{\prime}}\left(\overrightarrow{0}, t_{0}\right)\right| 0\right\rangle \\
& =\sum_{n}\left\langle 0\left|p_{\alpha}(\vec{x}) n_{\beta}(\vec{y})\right| n\right\rangle A_{n} e^{-E_{n}\left(t-t_{0}\right)},
\end{aligned}
$$

where $E_{n}$ denotes the energy of the state $|n\rangle, t_{0}$ the time-slice on which the source is located. $A_{n} \equiv\left\langle n\left|\bar{p}_{\alpha}(0) \bar{n}_{\beta}(0)\right| 0\right\rangle$ plays the role of an "overlap". Eq. (2.1) satisfies the Bethe-Salpeter (BS) equation, since it is obtained as a solution to the BS equation for the NN system in the BS framework. By using the procedure of the non-relativistic reduction, the BS equation reduces to the effective Schrödinger equation [4] as

$$
\left(\nabla^{2}+k^{2}\right) \phi(\vec{x} ; k)=m_{N} \int d^{3} y V_{k}(\vec{x}-\vec{y}) \phi(\vec{y} ; k)
$$

where the nucleon mass $m_{N}$ is introduced in the r.h.s. for later convenience. The interaction kernel $V_{k}(\vec{x}-\vec{y})$, in general, depends on $k$, which is the main reason why Eq. (2.3) is referred to as "effective" Schrödinger equation. At low energies, $V_{k}$ can be approximated as $V_{k}(\vec{x}-\vec{y}) \simeq V_{k \equiv 0}(\vec{x}-\vec{y})$. We are left with the Schrödinger equation for the NN system as

$$
-\frac{1}{2 \mu} \nabla^{2} \phi(\vec{x} ; k)+\int d^{3} y V_{N N}(\vec{x}-\vec{y}) \phi(\vec{y} ; k)=E \phi(\vec{x} ; k)
$$

where $\mu \equiv m_{N} / 2$ denotes the reduced mass of the nucleon, and $E \equiv k^{2} /(2 \mu)$ denotes the nonrelativistic "energy".

According to the standard nuclear physics [6], the NN interaction $V_{N N}$ is parameterized in the low energy region as

$$
\begin{aligned}
V_{N N} & =V_{\text {central }}(r)+V_{\text {tensor }}(r) \hat{S}_{12}+V_{\mathrm{LS}}(r) \hat{\vec{L}} \cdot \hat{\vec{S}}+O\left(p^{2}\right), \\
\hat{S}_{12} & \equiv 3 \frac{\left(\hat{\vec{\sigma}}_{p} \cdot \vec{x}\right)\left(\hat{\vec{\sigma}}_{n} \cdot \vec{x}\right)}{r^{2}}-\left(\hat{\vec{\sigma}}_{p} \cdot \hat{\vec{\sigma}}_{n}\right), \\
\hat{\vec{L}} & \equiv-i \vec{x} \times \vec{\nabla}, \quad \hat{\vec{S}} \equiv\left(\hat{\vec{\sigma}}_{p}+\hat{\vec{\sigma}}_{n}\right) / 2,
\end{aligned}
$$


where $r \equiv|\vec{x}|$ denotes the distance between the proton and the neutron. $\hat{\vec{\sigma}}_{p}$ and $\hat{\vec{\sigma}}_{n}$ denote the spin Pauli matrices for the proton and the neutron, respectively. $\hat{\vec{L}}$ represents the relative angular momentum operator of the proton from the neutron. $V_{\text {central }}(r)$ is referred to as the "central" NN force, which only depends on the distance $r$. It is considered to be the most important local interaction in the NN interaction. (Interactions which do not involve any derivatives are referred to as "local".) $V_{\text {tensor }}(r)$ is referred to as the "tensor" force, which is the second important local interaction. $V_{\mathrm{LS}}(r)$ is referred to as the "spin orbit interaction", which is the most important non-local term. Finally, $O\left(p^{2}\right)$ represents remaining non-local contributions, which is often considered to be less important.

Restriction to the $J^{P}=0^{+}$channel permits a further simplification. Because only the s-wave orbital component is allowed in this quantum number due to the parity selection rule, the contributions from the second and the third terms vanish. Since, then, $V_{N N}$ receives the contribution only from the central term as

$$
V_{N N} \simeq V_{\text {central }}(r)
$$

Eq. (2.4) reduces to

$$
-\frac{\vec{\nabla}^{2}}{2 \mu} \phi(\vec{x} ; k)+V_{\text {central }}(r) \phi(\vec{x} ; k)=E \phi(\vec{x} ; k) .
$$

Since $V_{\text {central }}(r)$ is a simple multiplication operator, we can rearrange it in the following way:

$$
V_{\text {central }}(r)=E+\frac{1}{2 \mu} \frac{\vec{\nabla}^{2} \phi(\vec{x} ; k)}{\phi(\vec{x} ; k)} .
$$

This relation states that, by identifying the BS wave function for NN, which is obtained from lattice QCD calculations, as the Schrödinger wave function, $V_{\text {central }}(r)$ can be reconstructed so that the Schrödinger equation is satisfied. It should be emphasized that, by construction, nothing is required on the quark mass to define the fixed separation between the two nucleons. Since the NN potential is reconstructed from the NN wave function, this method is expected to provide such NN potentials that are faithful to those obtained in the analysis of the $\mathrm{NN}$ scattering data.

\section{The numerical calculation}

We employ the standard Wilson gauge action at the gauge coupling $\beta=5.7$ on the $16^{3} \times 24$ lattice together with the standard Wilson quark action with the hopping parameter $\kappa=0.1665$. The lattice spacing is determined from $\rho$ meson mass in the chiral limit as $a^{-1}=1.44(2) \mathrm{GeV}$ $(a \simeq 0.137 \mathrm{fm})$. The physical volume of our lattice is $(2.2 \mathrm{fm})^{3} \times(3.3 \mathrm{fm})$. These parameters reproduces $m_{\pi} \simeq 0.53 \mathrm{GeV}, m_{\rho} \simeq 0.88 \mathrm{GeV}$, and $m_{N} \simeq 1.3 \mathrm{GeV}$. (See Ref. [7] for detail.) The global heat-bath algorithm is used to generate the gauge configurations. After skipping 5000 sweeps for thermalization, the gauge configurations are picked up every 100 sweeps. Totally, 160 gauge configurations are used for the measurement. Dirichlet boundary condition is imposed on the quark fields along the temporal direction on the time-slice $t=0$. To enhance the ground state contribution of the NN wave function, we adopt a spatially extended source with the gaussian smearing method with the size $\rho \simeq 0.4 \mathrm{fm}$ on the time-slice $t=5$. The BS wave function for $\mathrm{NN}$ is measured on the time-slice $t=10$, which is determined from a plateau appearing in the NN effective mass plot shown in Fig. (1). We keep in mind that the ground state saturation of the BS wave function is quite important. 


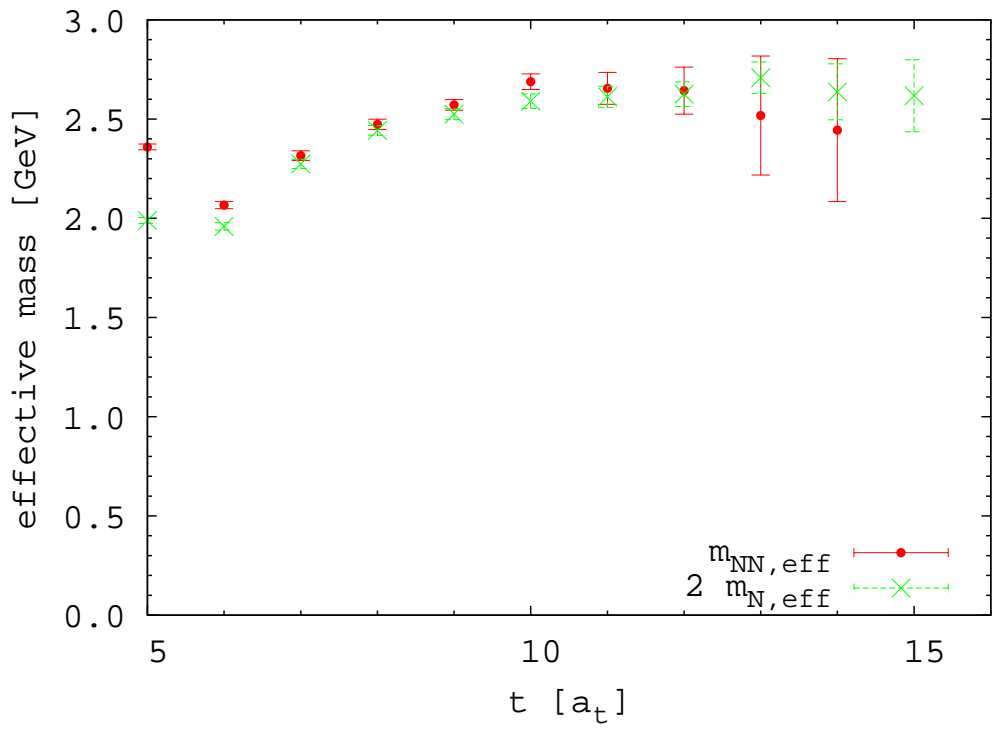

Figure 1: The effective mass plot. Dots denote the effective mass of the NN system $\left(J^{P}=0^{+}, I=1\right)$, whereas crosses denote twice the effective mass of the nucleon.

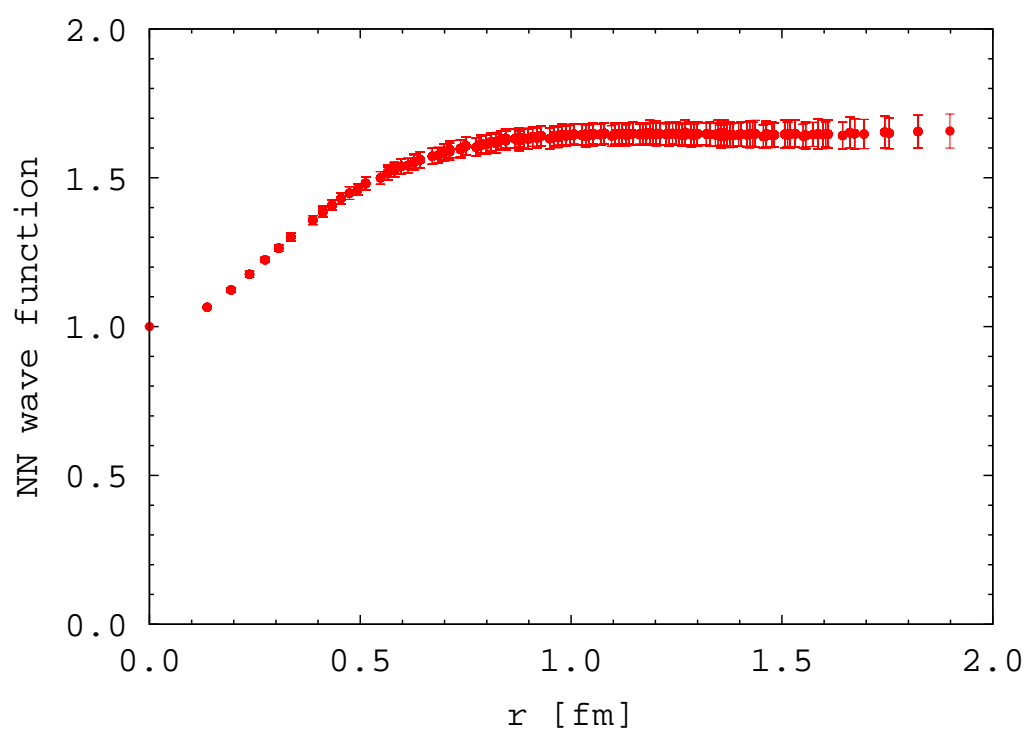

Figure 2: The lattice QCD result of the $\mathrm{NN}$ wave function $\left(J^{P}=0^{+}, I=1\right)$. 


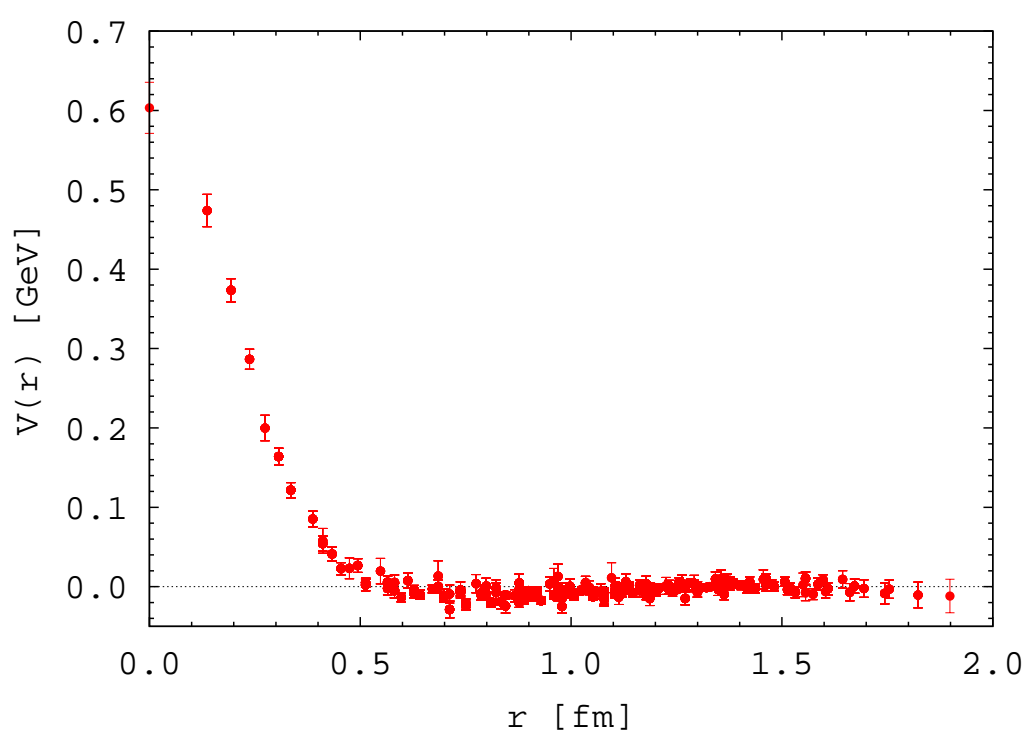

Figure 3: The lattice QCD result of the NN potential $V_{\text {central }}(r)$.

Fig. (2) shows the lattice QCD result of the BS wave function for NN. It is normalized with its value at the origin $\vec{r}=\overrightarrow{0}$. Not only the on-axis data, but also the off-axis data are plotted all together, due to which the horizontal axis extends beyond $L / 2 \sim 1.1 \mathrm{fm}$ to $\sqrt{3} L / 2 \sim 1.9 \mathrm{fm}$ ( $L \simeq 2.2 \mathrm{fm}$ is the spatial lattice extension). We observe that the wave function shrinks near the origin suggesting the existence of repulsion at short distance.

Fig. (3) shows the lattice QCD result of the NN potential $V_{\text {central }}(r)$ for $J^{P}=0^{+}, I=1$ channel. The simplest nearest neighbor numerical Laplacian for Eq. (2.8) is employed in reconstructing $V_{\text {center }}(r)$. The zero adjustment due to $E \equiv \frac{1}{2 \mu} k^{2}$ in Eq. (2.8) has not yet been performed because of the large noise. In principle, Lüscher's $k$ can be obtained from the difference of the effective

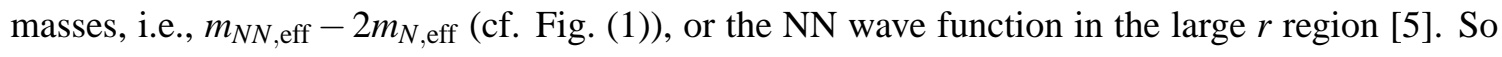
far, the data is too noisy to obtain it. We observe that, corresponding to the shrink in Fig. (2), $V_{\text {central }}(r)$ has a clear repulsive core of about $500 \mathrm{MeV}$ in the short distance region $r \lesssim 0.5 \mathrm{fm}$. On the other hand, we do not find a significant attraction in the intermediate distance region, which may be attributed to (i) the still large noise, (ii) finite volume effect ( $L \sim 2.2 \mathrm{fm}$ is too small for $\mathrm{NN})$, (iii) the large pion mass $\left(m_{\pi} \sim 0.53 \mathrm{GeV}\right)$. In order to gain physics insights into the origin of the repulsive core at short distance, we need more information such as the quark mass dependence, the flavor dependence, etc.

\section{Summary and discussion}

To study the nuclear force, we have applied a new method recently proposed by CP-PACS collaboration in the context of the $\pi \pi$ scattering phase shift. In this method, constraint on the quark mass to define the distance between the two nucleons is not necessary. Because the NN potential is reconstructed from the NN wave function, it is expected to provide NN potentials, which are faithful to those obtained from the analysis of the NN scattering data. By restricting ourselves to 
the quantum number $J^{P}=0^{+}$and $I=1$, we have reconstructed the central part of the NN-potential $V_{\text {central }}(r)$ from the Bethe-Salpeter wave function for NN obtained by using lattice QCD. We have seen that $V_{\text {central }}(r)$ has a clear repulsive core of about $500 \mathrm{MeV}$ in the short distance region $r \lesssim 0.5$ $\mathrm{fm}$. However, we have not found a significant attraction in the intermediate distance region, which may be attributed to the poor statistics, the small lattice volume $(L \sim 2.2 \mathrm{fm})$ and the large pion mass $m_{\pi} \sim 0.53 \mathrm{GeV}$. We are currently performing a lattice QCD calculation for $V_{\text {central }}(r)$ using a better statistics on a larger lattice volume with a lighter pion mass, which will be presented elsewhere. Our method have appeared to be quite promising in reproducing the NN potential with lattice QCD. It is interesting to apply our method to other channels to construct $V_{\mathrm{LS}}(r)$ and $V_{\text {tensor }}(r)$. Also, it is important to apply our method to hyperon-nucleon and hyperon-hyperon forces, where only a limited number of experimental data are available so far.

\section{Acknowledgments}

This research was partially supported by the Ministry of Education, Science, Sports and Culture, Grant-in-Aid for Scientific Research on Priority Areas No. 15540251 and No. 17070002, Scientific Research (C) No. 13135204 and No. 18540253. Lattice QCD Monte Carlo calculations have been performed with IBM Blue Gene/L at KEK under a support of its Large Scale Simulation Program.

\section{References}

[1] H. Yukawa, Proc. Math. Phys. Soc. Japan 17, 48 (1935).

M. Taketani, S. Nakamura, M. Sasaki, Prog. Theor. Phys. 6, 581 (1951).

P. Signell, Advances in Nucl. Phys. Vol. 2, 223 (1969), and references therein.

R. Machleidt, Advances in Nucl. Phys. Vol. 19,189 (1989), and references therein.

M. Oka, K. Yazaki, in Quarks and Nuclei, International Review of Nuclear Physics, Series, Vol. 1 (1984), edited by W. Weise (World Scientific, Singapore), p. 489, and references therein. F. Myhrer, J.Wroldsen, Rev. Mod. Phys.60, 629 (1988), and references therein.

[2] T.T. Takahashi, T. Doi, H. Suganuma, hep-lat/0601006.

T. Doi, T.T. Takahashi, H. Suganuma, hep-lat/0601008.

[3] A. Mihály, H.R. Fiebig, H. Markum, K. Rabitsch, Phys. Rev. D55, 3077 (1997);

C. Michael, P. Pennanen (UKQCD Collab.), Phys. Rev. D60, 054012 (1999).

[4] M. Lüscher, Commun. Math. Phys. 105, 153 (1986).

[5] CP-PACS Collab., S. Aoki et al., Phys. Rev. D71, 094504 (2005).

[6] P. Ring and P. Schuck, "The Nuclear Many-Body Problem", Springer-Verlag, p.1.

[7] M. Fukugita, Y. Kuramashi, M. Okawa, H. Mino, A. Ukawa, Phys. Rev. D52, 3003 (1995). 